\title{
Numerical Simulations of the Boundary Layer Transition Flight Experiment
}

\author{
Chun Y. Tang* and Kerry A. Trumble ${ }^{\dagger}$ \\ NASA Ames Research Center, Moffett Field, CA \\ Charles H. Campbell ${ }^{\star}$ \\ NASA Johnson Space Center, Houston, TX \\ Victor R. Lessard ${ }^{\S}$ and William A. Wood ${ }^{* *}$ \\ NASA Langley Research Center, Hampton, VA
}

\begin{abstract}
Computational Fluid Dynamics (CFD) simulations were used to study the possible effects that the Boundary Layer Transition (BLT) Flight Experiments may have on the heating environment of the Space Shuttle during its entry to Earth. To investigate this issue, hypersonic calculations using the Data-Parallel Line Relaxation (DPLR) and Langley Aerothermodynamic Upwind Relaxation ( LAURA) CFD codes were computed for a 0.75" tall protuberance at flight conditions of Mach 15 and 18. These initial results showed high surface heating on the BLT trip and the areas surrounding the protuberance. Since the predicted peak heating rates would exceed the thermal limits of the materials selected to construct the BLT trip, many changes to the geometry were attempted in order to reduce the surface heat flux. The following paper describes the various geometry revisions and the resulting heating environments predicted by the CFD codes.
\end{abstract}

\section{Nomenclature}

$\begin{array}{ll}B F & =\text { Bump factor, a normalized heat flux } \\ \text { BLT } & =\text { Boundary layer transition } \\ \text { CFD } & =\text { Computational fluid dynamics } \\ \text { DPLR } & =\text { Data parallel line relaxation CFD code } \\ \text { LAURA } & =\text { Langley aerothermodynamics upwind relaxation algorithm CFD code } \\ \mathrm{M} & =\text { Mach number } \\ \mathrm{OML} & =\text { Outer mold line } \\ \mathrm{RCG} & =\text { Reaction cured glass } \\ \mathrm{STS} & =\text { Space transportation system } \\ \mathrm{TPS} & =\text { Thermal protection system }\end{array}$

\section{Introduction}

$\mathrm{T}$ he Boundary Layer Transition (BLT) Flight Experiment ${ }^{1}$ was designed to validate turbulent Computational Fluid Dynamics (CFD) simulations against hypersonic flight data for the Space Shuttle Orbiter. As discussed in reference 2, previous turbulent flight data was limited to two thermocouple measurements obtained from STS-28, in which the flow transitioned, earlier than expected, to turbulent at approximately Mach 16. Thus, one primary objective of the BLT flight experiment was to induce early flow transition and to gather data on and downstream of the protuberance. This flight data is important for validating CFD solutions, engineering models, and ground-based

\footnotetext{
* Senior Research Scientist, Aerothermodynamics Branch, MS 230-2, AIAA member.

${ }^{\dagger}$ Research Scientist, Aerothermodynamics Branch, MS 230-2, AIAA member.

* Aerospace Engineer, Applied Aeroscience and CFD Branch, M/C EG3, Senior AIAA member.

$\S$ Engineer, Gentex Systems, MS 408A, Senior AIAA member.

** Aerospace Engineer, Aerothermodynamics Branch, Senior lifetime AIAA member.
} 
test data. As seen in Fig. 1, the initial design of the BLT trip was a protrusion that measured 4.0 inches in length and 0.75 inches in height. The protuberance was located on the windward side of Orbiter's port wing (see Fig. 2). This location was chosen based on structural and thermal considerations, as well as accessibility of instrumenting thermocouples on the heat tiles downstream of the protuberance. Since it was expected that the presence of a protrusion would induce early flow transition and result in augmented heating around the trip, a series of CFD simulations were computed to estimate the local heating environment. These solutions were then used in designing the BLT protuberance and to assess possible safety concerns associated with the installation of a protruding element on the tile surface of the Orbiter.

\section{Computational Methodology}

Aerothermodynamic analyses of the BLT protuberances are computed using the Data-Parallel Line Relaxation $(D P L R)^{3}$ and the Langley Aerothermodynamic Upwind Relaxation Algorithm (LAURA $)^{4,5}$ CFD codes. DPLR is a parallel, multi-block, finite volume code that solves the reacting Navier-Stokes equations including finite rate chemistry and thermal non-equilibrium. LAURA is a three-dimensional, second-order finite volume flow solver for non-equilibrium chemistry. These cases are modeled using a five species $\left(\mathrm{N}, \mathrm{O}, \mathrm{N}_{2}, \mathrm{O}_{2}\right.$, and NO) chemical nonequilibrium, non-ionizing air chemistry $\operatorname{model}^{6}$. A radiative equilibrium wall temperature boundary condition is set at the surface, and Reaction Cured Glass (RCG) catalycity ${ }^{7}$ are applied at the surface of the Orbiter tiles and the BLT protuberance. Both codes have been used extensively to predict aerothermal environments for the Space Shuttle as well as other Earth entry vehicles (e.g., Fire-II' ${ }^{8}$, Apollo ${ }^{9,10}$, and Genesis ${ }^{11,12}$ ).

\section{A. Protuberance modeling}

To simplify the grid generation process, a 0.06 inches fillet at the base of the trip has been neglected in our simulations. For the initial BLT calculations, rapid analysis procedures ${ }^{13}$ developed to analyze damages sustained during a Shuttle mission were modified to setup the protuberance simulations. The methodology of the rapid analysis procedures is to reduce the computational domain to a small region around the geometric feature. These "local" simulations allow for faster turnaround times by reducing the complexity of generating a point-matched structured grid over the entire vehicle and by lowering the total number of grid points (hence, the CPU time) in the simulations. Since $D P L R$ and LAURA use different procedures to run these local simulations, the details of both processes are outlined below.

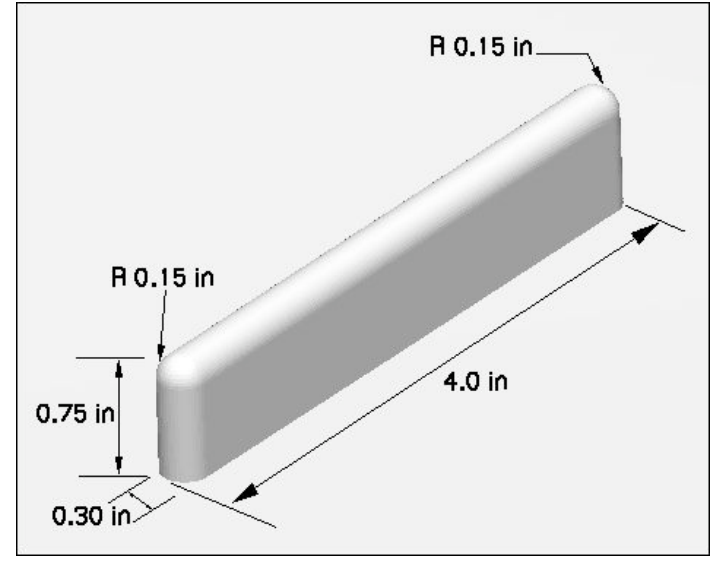

Figure 1. Model of BLT protuberance.

\section{B. DPLR simulations}

Within DPLR, the best practices for modeling surface anomalies on the Shuttle is to use a local analysis approach, where an existing baseline solution for an undamaged Orbiter is interpolated and applied as boundary conditions on a local grid. The main advantages of running a local simulation are faster turnaround times and simplification of the grid generation procedure. The grid generation process starts by creating a surface mesh on the BLT geometry and generating a local surface grid around the base of the protuberance using a hyperbolic surface extrusion algorithm available in the commercial code Gridgen ${ }^{14}$. These surfaces grids are projected onto the Orbiter database so they would match the Shuttle geometry in the baseline solutions. Volume blocks surrounding the protuberance are then created via a normal volume extrusion algorithm in Gridgen. In order to connect these volumes grid to the outer boundary of the baseline solution, the RADIAL_INTERP $P^{15}$ code is utilized. This extended volume is then combined with the volume grid surrounding the protuberance to create the overall computational domain. This approach offers the flexibility of not requiring the local mesh to adhere to the baseline grid topology; therefore, the grid topology and mesh dimensions can be tailored to the protuberance geometry. Since the outer boundary of each baseline solution is adapted to the specific bow shock location of the freestream condition, the extended volume grid is generated separately for each Mach number simulation. The volume blocks surrounding the protuberance, however, can be reused for all cases as long as the protrusion geometry is unchanged.

The starting solution for each case is obtained using the FLOW_INTERP ${ }^{16}$ utility. This code interpolates the smooth Outer Mold Line (OML) solution onto the new local volume grid. The solutions at the far-field boundaries are imposed as either fixed inflow boundary conditions (if the flow is entering the volume) or supersonic outflow 
boundary conditions (if the flow is exiting the volume). For more details, see $\mathrm{BC}=2$ in the DPLR user manual ${ }^{17}$. Using an interpolated baseline solution as an initial guess also improves the convergence of these BLT computations. It should be noted that all DPLR simulations are computed as steady state; therefore, the residuals may not reach machine zero if the flowfield is unsteady (e.g., separated flow behind the trip). The solution is deemed converged if the differences in the surface heat flux between iterations are less than a user specified criteria.

Overall, the local approach is a faster way to obtain a solution since the simulation has fewer overall grid points than an equivalent full body simulation with the same level of detail. Fig. 2 shows the location and physical size of the local protuberance grid with respect to the Shuttle geometry. As evident in the figure, the domain of the local simulation is much smaller than the original baseline grid. If the BLT protuberance calculations were computed over the entire Shuttle, the CPU time and memory requirements would likely increase by an order of magnitude.

\section{LAURA simulations}
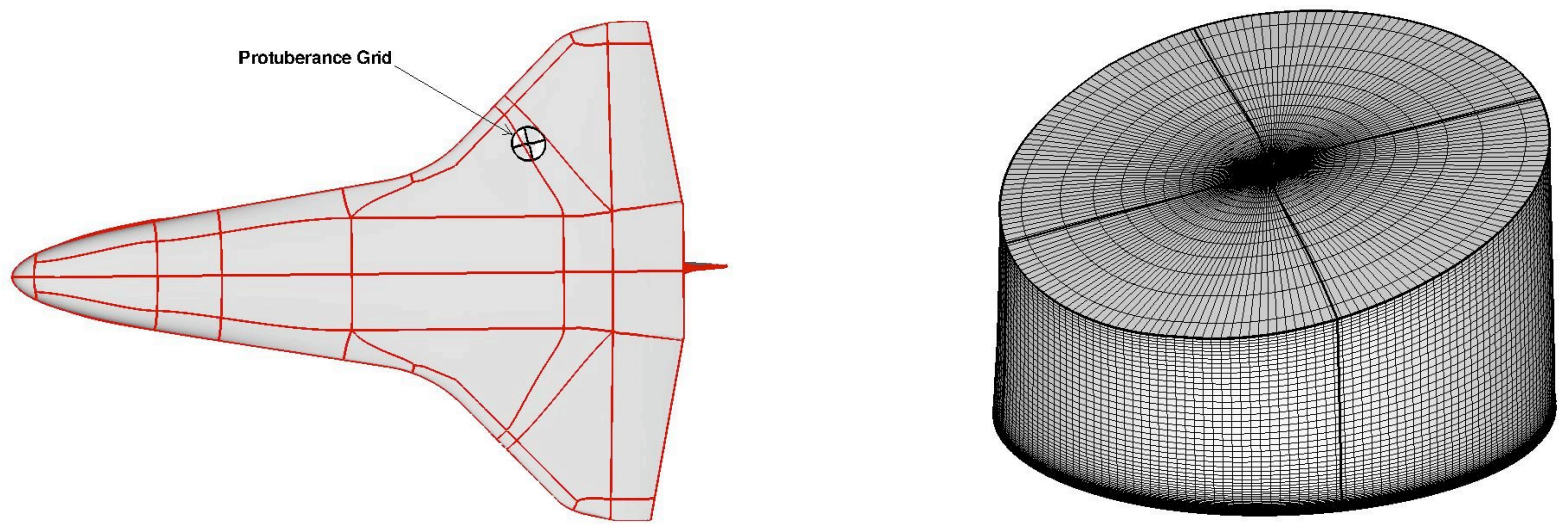

Figure 2. Location of BLT protuberance (left) and close-up of local volume grid (right).

Modeling the trip device on the full Orbiter using LAURA is a significant effort and is beyond the scope of the present work. The problem is reduced by leveraging existing LAURA solutions for the Orbiter at the stated flight conditions and using a similar approach that NASA Langley's $M O R P H^{18}$ code performs without the automated grid generation process. The MORPH software is not utilized directly due to the shape of the trip device. The spherical top corners and rounded edges of the trip are not ideal for using MORPH because of the highly skewed cells that are created in the grid at these locations. Hence, a manual approach is used to generate the trip grid system using a process similar to MORPH of extracting the surrounding or bounding grid blocks from the existing LAURA solution
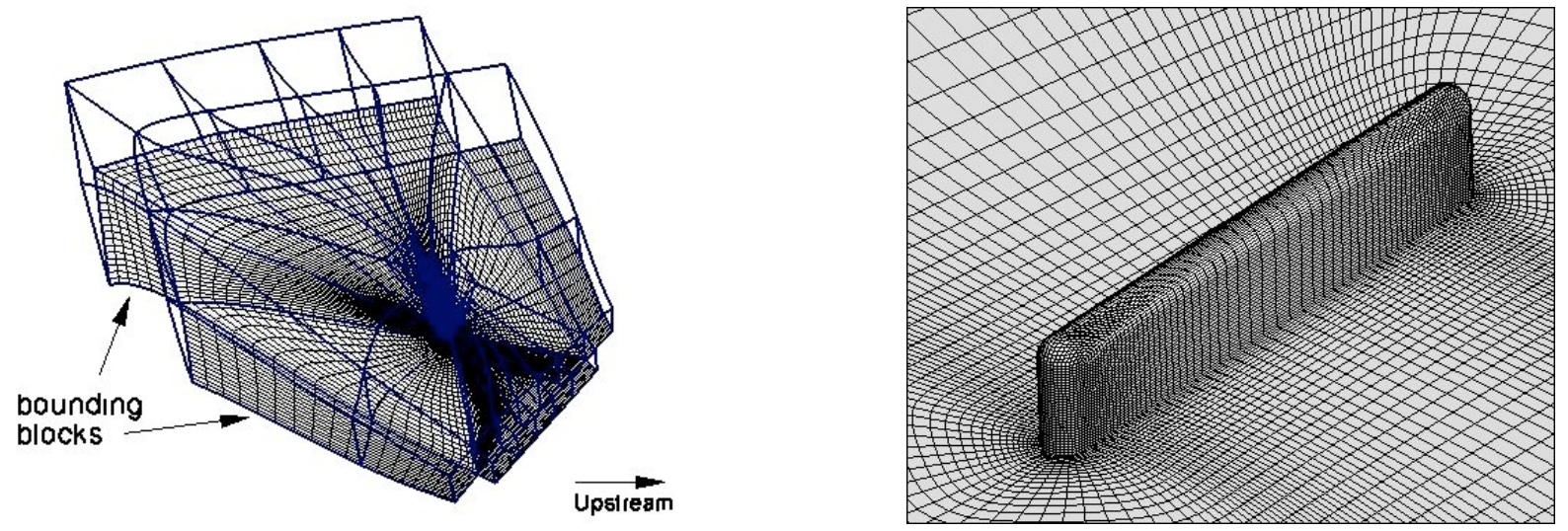

Figure 3. Local mesh with bounding and interior grid blocks (left) and surface grid around the trip (right).

in the region of the trip location. New interior grid blocks are created encompassing the trip device using Gridgen. The new interior grid blocks are connected to the extracted bounding blocks using a one-to-one point grid connection. The interior grid blocks' topology is such that the Orbiter's surface and the trip surface are at a grid 
index of $\mathrm{k}=1$ plane. Essentially, the $\mathrm{k}=1$ plane wraps around the entire BLT trip. The entire new grid system contains 52 blocks, where 14 of them are bounding blocks and 38 are interior blocks surrounding the trip. The original number of grid points (being 65) in the k-direction, extending from the surface to the outer boundary, is maintained in the new grid system. Shown in Fig. 3 is a plot of the local volume grid and the surface grid around the trip.

The existing LAURA solution, for each given flight case, is interpolated onto the new grid system and used as a starting guess for the trip analysis. Similar to the DPLR simulations, the grid generation and solution extraction process is repeated for each case due to the fact that the existing LAURA solutions have different grids tailored to the bow shock position.

\section{Comparison of grids}

Because the solutions between DPLR and LAURA are compared, it is important to examine grid differences used for each solver. Fig. 4 compares the surface grids on and around the trip for the coarse DPLR grid (shown as black) versus the nominal LAURA grid (shown as red). Clearly, the meshes differ in topology as well as grid density. By design, the LAURA mesh had nearly orthogonal grids normal to the surface of the trip. The DPLR grid has more skewed grid cells at the top of the protuberance since it used an automated grid generation process (developed for modeling protruding gap fillers with sharp corners at the top) to create the volume grid. As a result, some highly skewed grid cells were created at the rounded spherical cap at the top of the trip. To study grid resolution effects, a fine $D P L R$ grid with four times as many grid points on the surface of the protuberance while maintaining the same number of surface normal points was generated and used in a couple DPLR simulations.

\section{Results}
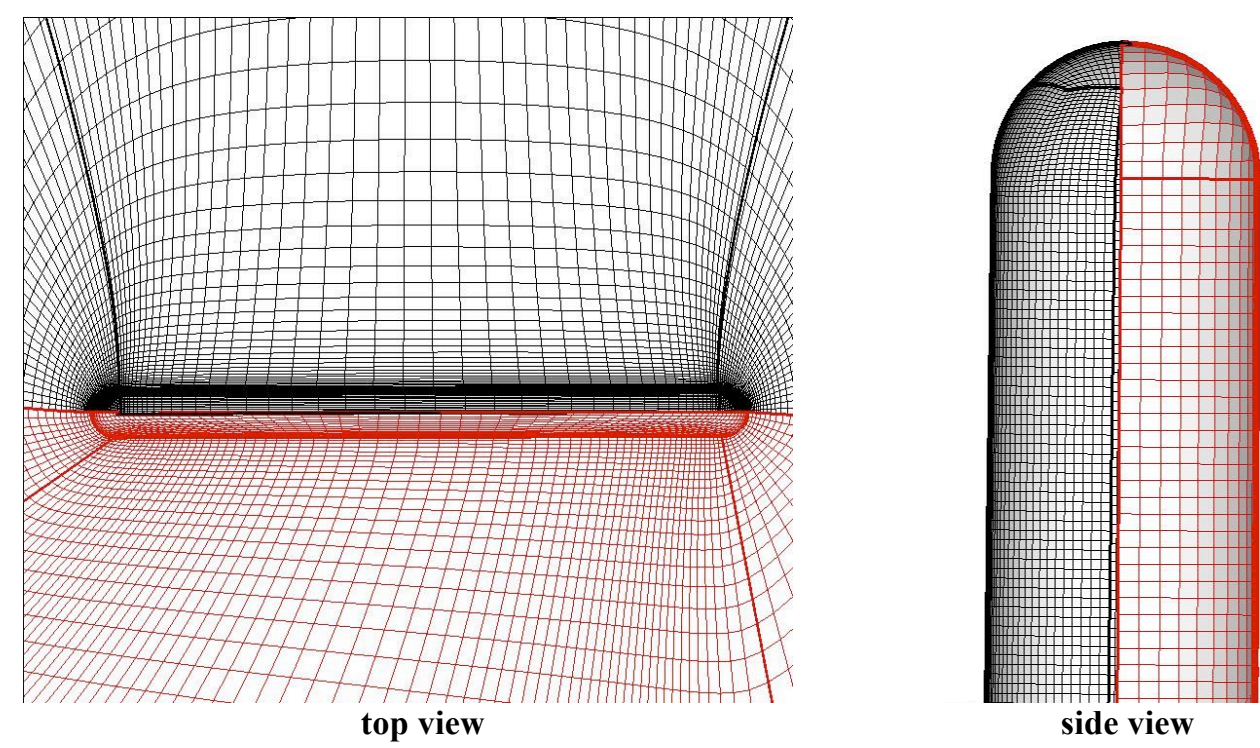

side view

Figure 4. Comparison of coarse $D P L R$ grid (black) vs. $L A U R A$ grid (red) at the trip surface.

\section{A. DPLR solutions}

In general, the DPLR simulation of the BLT trip did not converge to a steady state value (possibly due to the flow separating behind the protuberance). The solution was assumed to have converged if the maximum differences in surface heat flux was less than $+/-0.09 \mathrm{BTU} / \mathrm{ft}^{2}-\mathrm{s}$ between iterations. The streamlines in Fig. 5 illustrate a bow shock forming ahead of the protuberance and separated flow behind the trip. By design, the BLT trip is angled at approximately 45 degrees with the incoming streamlines. Hence, the stagnation point is not at the symmetry plane of the protuberance. To better visualize the heating differences, heating "bump factors" (BF) are calculated by normalizing the surface heat flux with a constant reference value. This reference value is usually selected as the heating rate of the baseline Orbiter solution at the location just upstream of the protuberance. Therefore, a bump factor greater than 1.0 indicates heating augmentation due to the presence of the trip. It should be noted that the heat flux does vary spatially on the baseline solution; thus, the bump factor may deviate slightly from a value of 1.0 
(typically between $0.9-1.1$ ) even for the baseline smooth OML solution at the protuberance location. Shown in Fig. 6 is a comparison of the heating BF contours on the trip at Mach 15 (left) and Mach 18 (right). We can clearly see a line of high heating along the top of the protrusion. This feature is probably a numerical artifact caused by the
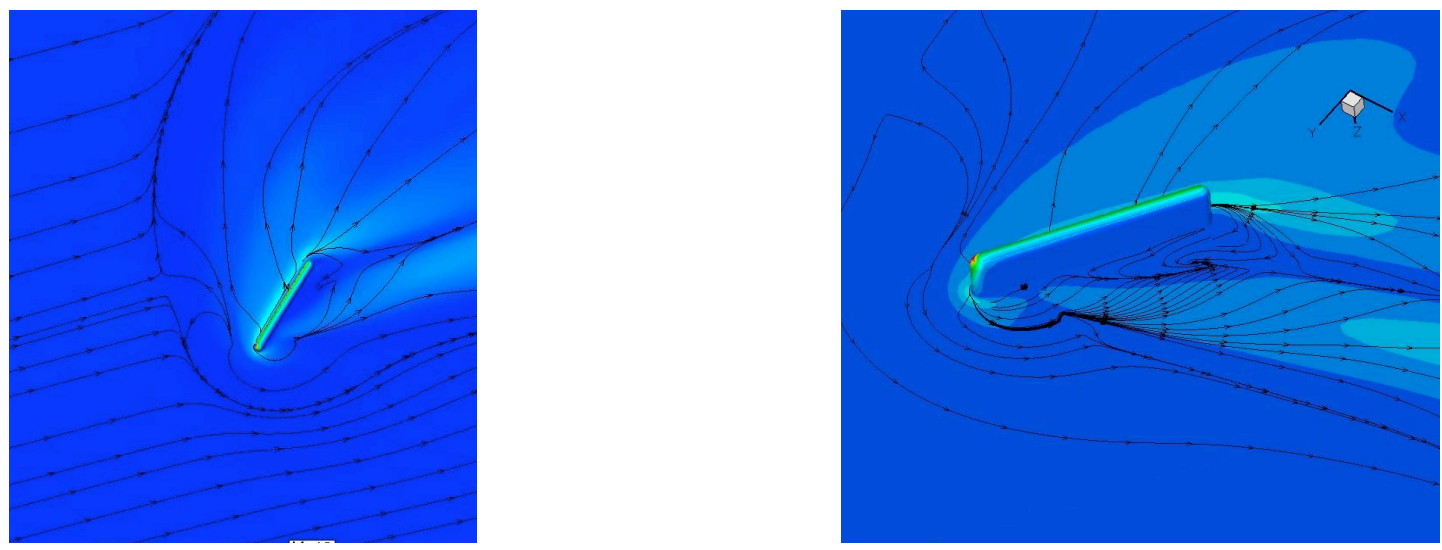

Figure 5. Streamlines around the BLT protuberance (Mach 18)

highly skewed grid cells located in this region. For the Mach 15 solution, the peak bump factor at the "nose" of the trip is around 55, which suggests that the peak heating on the protuberance is approximately 55 times higher than the smooth OML value. The Mach 18 solution has a slightly lower maximum BF value of 52 on the trip; however, the overall peak heating rate at Mach 18 is greater than at Mach 15 because the reference heat flux value is larger at the Mach 18 condition. Clearly, these simulations show significant heating at the nose of the trip and the tile area around the protuberance.

\section{B. LAURA solutions}

For the LAURA solutions, the convergence criterion was less than $1 \%$ difference in heating over 10,000
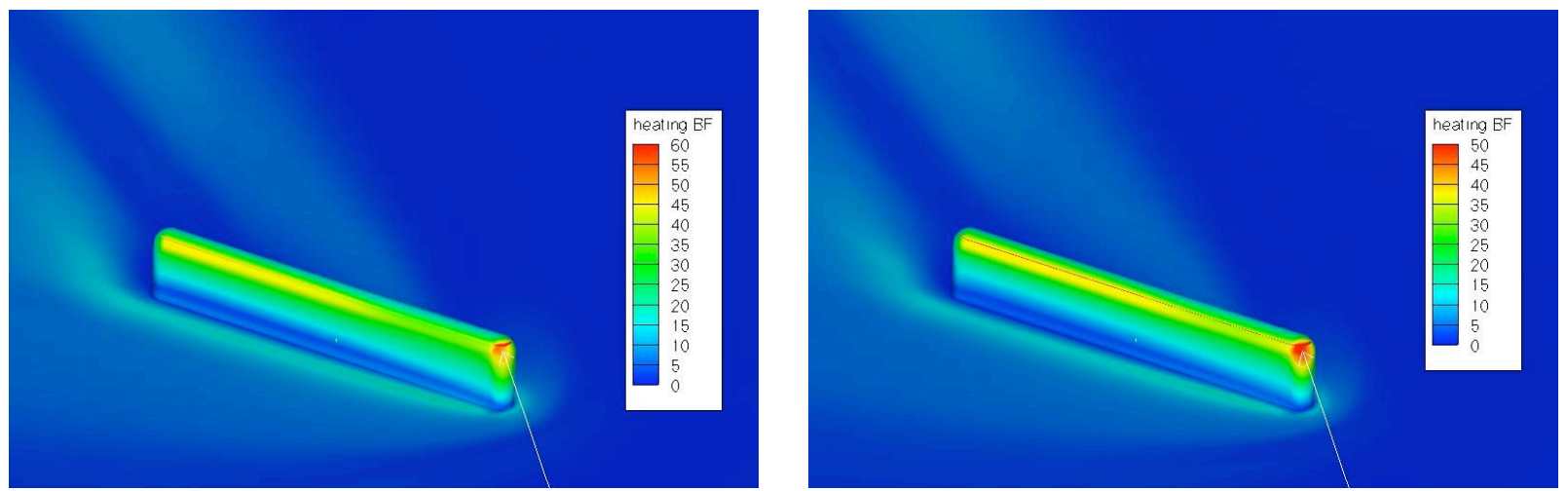

Figure 6. Comparison of $D P L R$ heating bump factor contours at Mach 15 (left) and Mach 18 (right)

iterations. The L-2 norm of the residual was less than $10^{-6}$, and the difference in surface temperature over 500 iterations was less than $0.2^{\circ} \mathrm{F}$. Shown in Fig. 7 is a comparison of the LAURA and DPLR heating BF contours at Mach 15. A similar plot at the Mach 18 condition is shown in Fig. 8. From these plots, we can see that the lines of high heating along the top of the protuberance, seen in the DPLR solutions, are not present in the LAURA results. In general, the maximum bump factors (hence, the heat flux) on the protuberance are higher in the $L A U R A$ solutions than the corresponding $D P L R$ results. Meanwhile, the peak bump factors in the acreage area are quite similar between the two solvers. It is interesting to note that the location of the stagnation point on the protuberance is slightly different between the two codes. One possible explanation is that the high skewness near the stagnation region in the DPLR grids is causing the peak heating point to shift to the side of the nose cap whereas the peak heating location in the LAURA solution is more forward on the nose cap. This difference in the stagnation location may account for some of the discrepancies in the peak BF on the protuberance predicted by the two solvers. For the 
surface heating on the acreage area, where both grids are fairly orthogonal, the two codes produced similar heating rates. There are some slight differences between the LAURA and DPLR solutions in the region behind the trip, where the flow is separated. It is likely that these discrepancies are caused by the difference in grid density between the LAURA and DPLR meshes. Typically, the behavior of separated flow in CFD calculations is quite sensitive to the density and skewness of the grid.

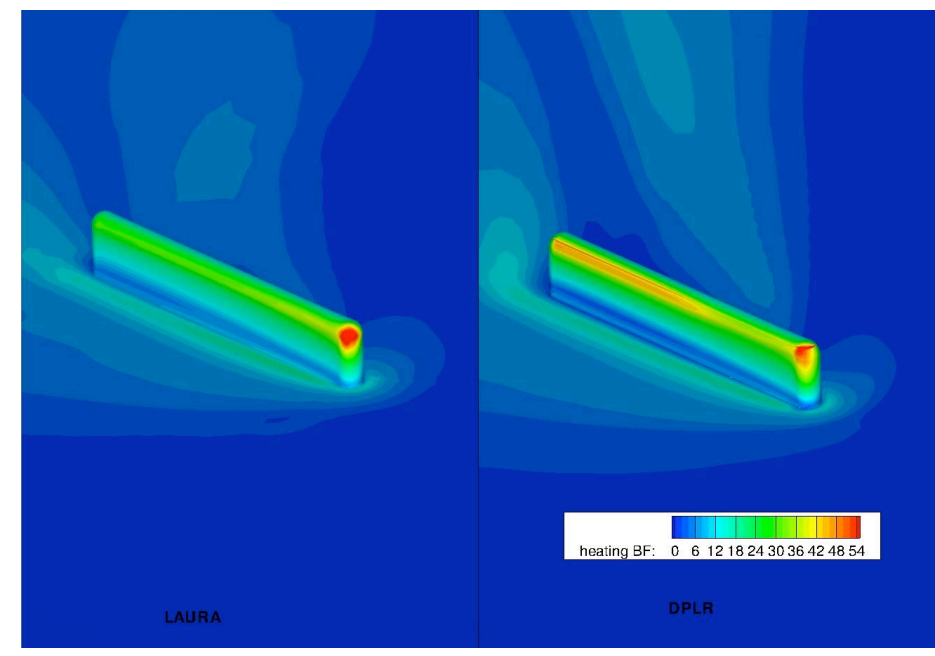

Figure 7. Comparison of bump factor contours between LAURA (left) and DPLR (right) at Mach 15.

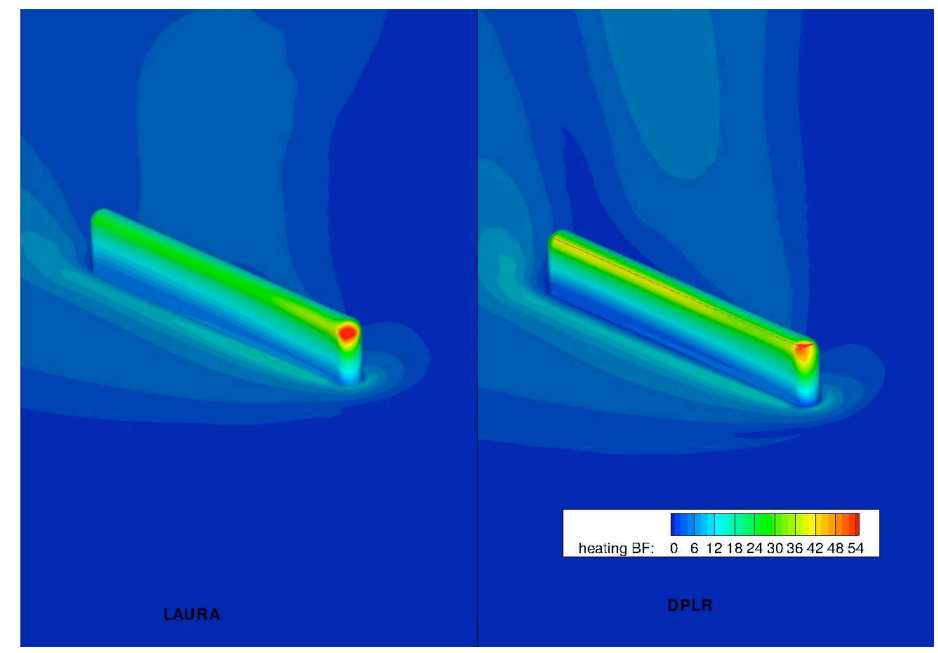

Figure 8. Comparison of bump factor contours between LAURA (left) and DPLR (right) at Mach 18.

To summarize the CFD results, Table 1 lists the maximum BF identified on the protuberance and on the acreage area. The table also includes $D P L R$ solutions computed on a fine grid (to study grid resolution effects) and for protuberance heights of 0.5 " and 0.25 ". Looking at the table, we can see that the fine DPLR grid resulted in a slightly higher BF on the protuberance while the heating rate was unchanged on the acreage. A closer look at the fine grid solution shows that the stagnation point has shifted slightly more forward of the nose cap (as compared to the coarse grid solution). This stagnation location is in better agreement with the LAURA prediction. It is also worthwhile to note that the relationship between bump factor and trip height appears to be fairly linear. Thus, one key design parameter in reducing heating rates on the protuberance and the acreage is to lower the protrusion height. 


\begin{tabular}{|l|c|c|c|c|}
\hline & \multicolumn{2}{|c|}{ Max protuberance BF } & \multicolumn{2}{c|}{ Max acreage BF } \\
\hline & DPLR & LAURA & DPLR & LAURA \\
\hline Mach 15, 0.75" trip & 55 & 67 & 18 & 19 \\
\hline Mach 18, 0.75" trip & $\begin{array}{c}52 \text { (coarse grid) } \\
59 \text { (fine grid) }\end{array}$ & 62 & $\begin{array}{c}15 \text { (coarse grid) } \\
15 \text { (fine grid) }\end{array}$ & 16 \\
\hline Mach 18, 0.5" trip & 30 & & 10 & \\
\hline Mach 18, 0.25" trip & 13 & & 5.3 & \\
\hline
\end{tabular}

Table 1. Comparison of peak heating bump factor values.

\section{Geometry Revisions}

To avoid the difficulties of certifying a new Thermal Protection System (TPS) material for the Space Shuttle, the BLT protuberance was constructed out of materials with properties similar to Reaction Cured Glass. Consequently, the maximum allowable bump factor at Mach 18 (using the baseline smooth OML heating rate at the trip location) is approximately 8. As we can see from Table 1, all of the predicted bump factors exceed this requirement; thus, a series of geometry changes were modeled numerically to help reduce the peak heating rates on the trip and the area surrounding it. For comparison purposes, all geometry revisions were computed at the peak heating condition of Mach 18.
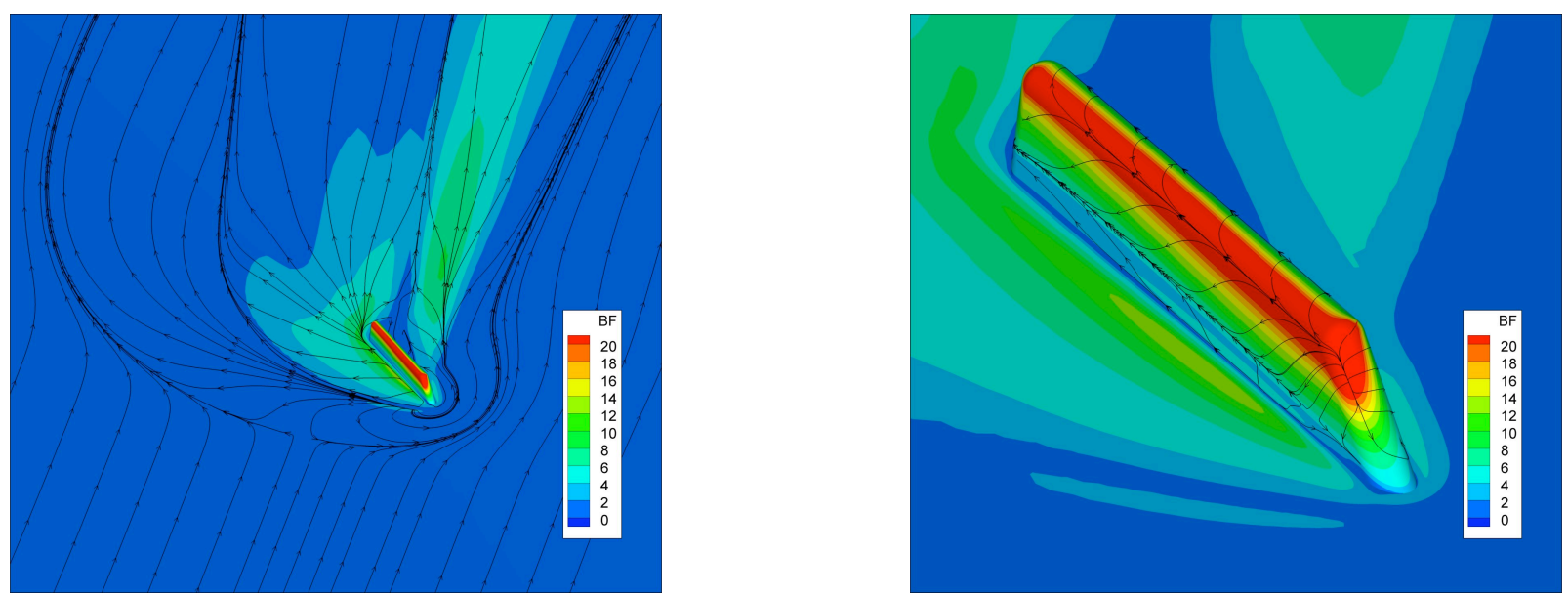

Figure 9. Surface heating BF contours for the Revision \#1 (height = 0.75").
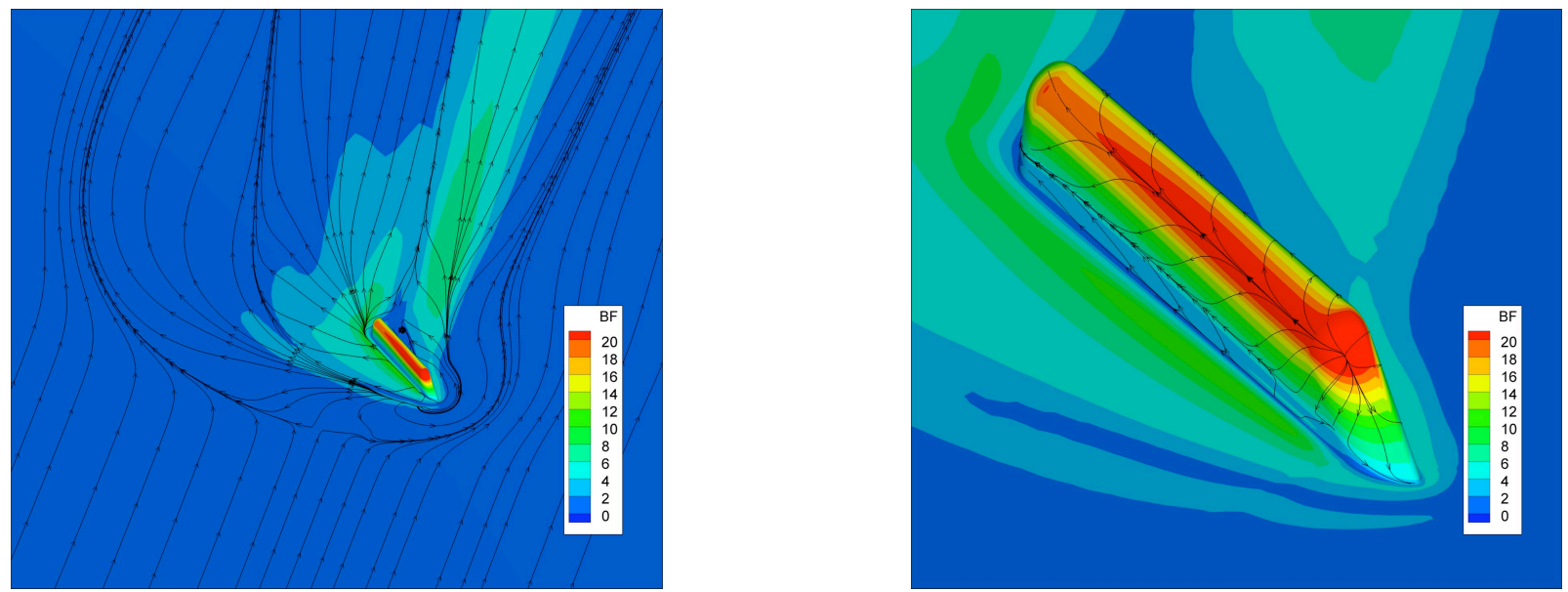

Figure 10. Surface heating BF contours for the Revision \#2 (height $=0.75$ ”).

Revision \#1 consisted of sweeping the leading edge of the baseline BLT protuberance by 45 degrees while maintaining a trip height of 0.75 " and a "clock" angle (angle between the centerline of the trip with the incoming 
local streamlines) of 45 degrees. The idea is similar to sweeping the wing of a supersonic aircrafts in order to reduce the intensity of the shock. Shown in Fig. 9 are contour plots of the surface heating on the protuberance and the acreage area surrounding the trip. The peak BF at the front of the trip is approximately 43 compared with 59 on the baseline configuration. Thus, the peak BF was reduced by $27 \%$ as a result of sweeping the protuberance. The swept trip had a smaller effect on the acreage heating. The peak acreage BF value was lowered to about 14 from the original value of 15 , which suggests that the acreage heating is more a function of the height rather than the shape of the trip.

Since the peak heating on Revision \#1 was still quite high, a second modification, Revision \# 2, was computed. This configuration is asymmetric in that the radius at the top of the trip (side facing the flow) was increased from 0.15 " to 0.3 " while maintaining the 45 degree sweep angle and the 45 deg clock angle. Fig. 10 shows the surface heating contours of the protuberance along with the heating environment on the acreage. The peak heating BF is approximately 32 at the front of the trip and about 11 on the acreage. Since surface heating on a sphere is inversely proportional to the square root of the sphere's radius, we would expect the heating on this revision to be reduced by a factor of $\sqrt{2}(\sim 1.414)$. For the Revision \#1 geometry, the peak BF on the protuberance was 43 . Therefore, we would expect the increased radius to reduce the BF to around $30.4(43 / 1.414)$, which is close to the value of 32 as predicted by CFD. One possible explanation for the slight discrepancy is due to a change in grid topology between Revisions \#1 and \#2. Revision \#2 has a grid topology that is similar to the one used in the LAURA simulations in order to avoid high grid skewness at the top of the trip. With this grid topology, the peak heating location at the nose of the protuberance is shifted more forward, which tends to yield a slightly higher heating rate. It should be noted that all subsequent BLT revisions ( $\# 3$ to $\# 8$ b) used a similar grid topology to reduce grid skewness at the top of the protuberance.

In another attempt to reduce the heating on the BLT protuberance, a Revision \#3 configuration with a radius of 0.6 ", a 60 deg. sweep angle, and a clock angle of $30 \mathrm{deg}$. was computed. Shown in Fig. 11 are heating bump factor contours for the BLT trip and the acreage area. The BF is approximately 36 at the front of the protuberance and 10 on the acreage area. It appears that the change in clock angle helped to lower the heating on the acreage region. However, the geometry changes also moved the peak heating location closer to the smaller radius at the top of the protuberance. Consequently, the peak heating in this revision was actually higher than the maximum value in Revision \#2.
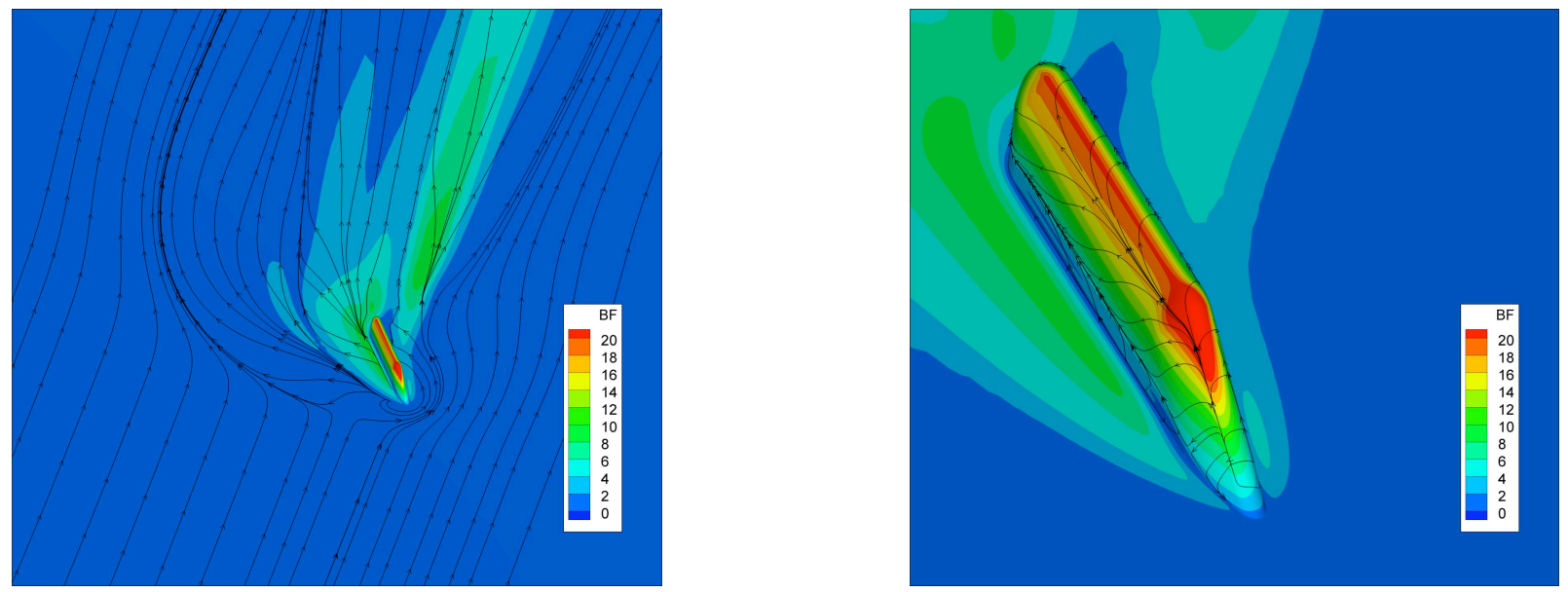

Figure 11. Surface heating BF contours for the Revision \#3 (height $=0.75$ ”).

Since the computed heat flux from the three geometry revisions are still significantly higher than the desired value, one way to drastically lower the heating rates is to reduce the height of the protuberance. Shown in Fig. 12 are surface BF contours for Revision \#5, a protuberance with a height of 0.5 ", $45 \mathrm{deg}$. sweep angle, $45 \mathrm{deg}$. clock angle, radius $=0.8 "$, and a fillet radius at the top $=0.06 "$. The surface heat flux is approximately 13 at the front and 7.4 on the acreage. A closer examination of surface streamlines on the trip shows that the peak heating is located at the top of the protuberance (near the fillet radius) rather than at the stagnation region near the nose of the trip. One possible explanation for this behavior is that the boundary layer gets thinner as the flow goes over the smaller radius at the top of the trip. This thinning of the boundary layer can result in higher surface heating.

Thus, the radius at the top of the BLT trip was increased in an attempt to lower the surface heat flux. Revision \#6 is a protuberance with a height of $0.5 ", 45 \mathrm{deg}$. sweep angle, $45 \mathrm{deg}$. clock angle, radius $=0.7$ ", and a fillet radius 
at the top $=0.12$ ". Plots of the heating bump factor contours (see Fig. 13) show values of 14 at the front and 7.1 on the acreage. These heating rates are very similar to those observed in Revision \#5, and the differences in heat flux between Revisions \#5 and \#6 are within the uncertainties of the CFD simulations. In terms of the heating environment, Revisions $\# 5$ and $\# 6$ are quite comparable. To reduce the heating even further, two Revisions (\#7b
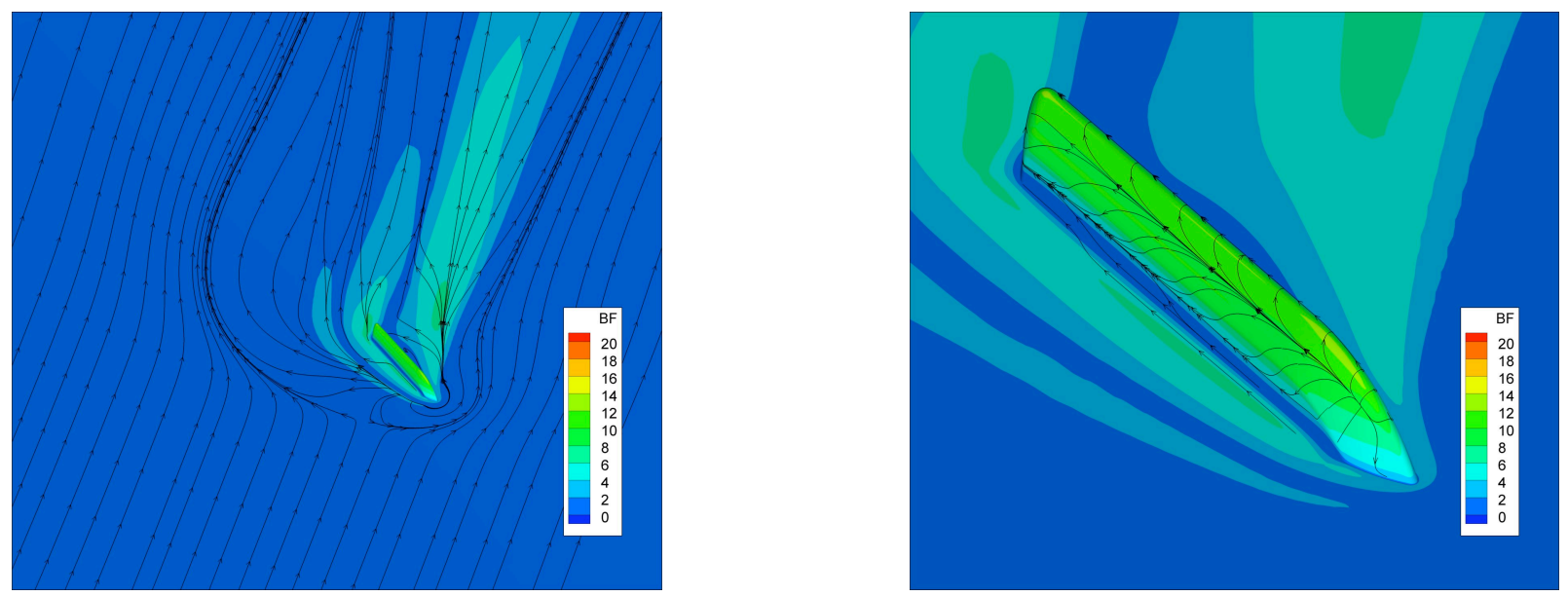

Figure 12. Surface heating BF contours for the Revision \#5 (height $=0.5$ ").

and \#8b) with protuberance heights of 0.35 " were simulated at the peak heating condition of Mach 18. In addition to having a lower height, both revisions also have larger radii of curvature at the top of the protuberance. Shown in Figs. 14 and 15 are plots of the heating BF contours for Revision \#7b and Revision \#8b, respectively. As evident from the graphs, the heating rates between Revisions $\# 7 \mathrm{~b}$ and $\# 8 \mathrm{~b}$ are approximately the same (maximum bump
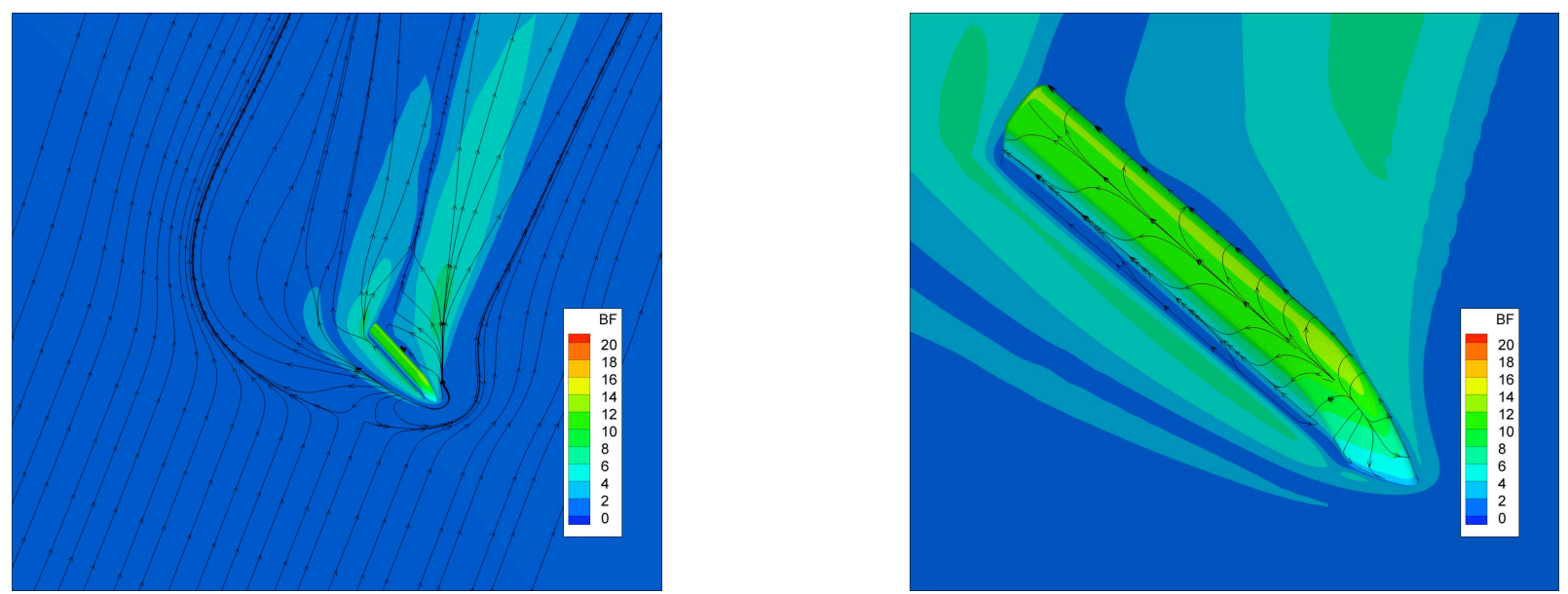

Figure 13. Surface heating BF contours for the Revision \#6 (height = 0.5”).

factors of $\sim 9$ ) on the protuberance while Revision \#8b has a slightly higher peak acreage BF.

Since the design goal is to have a peak $\mathrm{BF}$ value $<8$ on the protuberance, a simulation of a Revision $\# 7 \mathrm{~b}$ trip at a height of 0.25 " was computed. The bump factor contours for this case are shown in Fig. 16, and the solution predicts a peak BF value of approximately 6.8 on the protrusion and 5.2 on the acreage. Finally, the desired heating rates are achieved by altering the shape of the protuberance and by reducing its height to 0.25 ". Table 2 summarizes the bump factors associated with the different design changes.

Based on these CFD calculations, the first Boundary Layer Transition flight experiment was designed using a Revision \#7b configuration and a trip height of 0.25 ". This experiment flew on STS-119, which was launched on March 15, 2009. The validation of the CFD predictions with flight data is currently underway. A second flight experiment with a protuberance height of 0.35 " flew aboard STS-128, which was launched on August 28, 2009. Flight data from both BLT experiments are currently being used in reconstructing the thermal response of the protuberance and the heating augmentation downstream of the trip. 

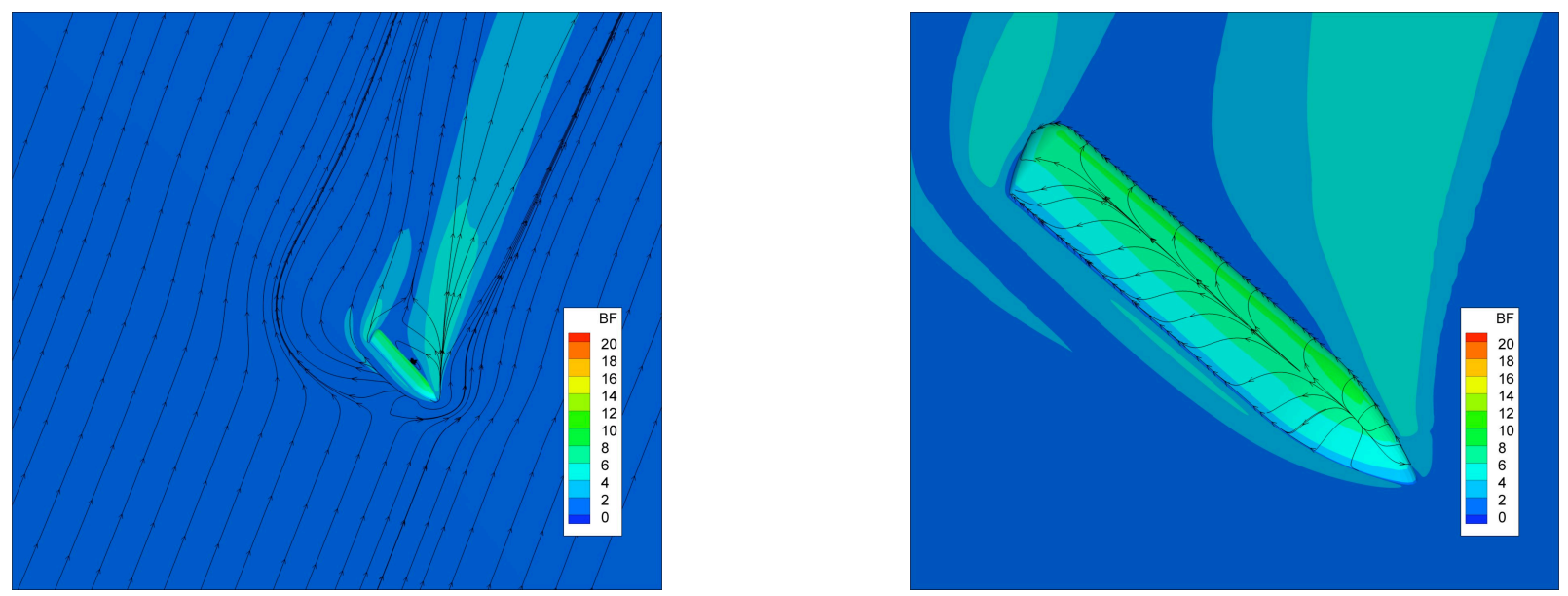

Figure 14. Surface heating BF contours for the Revision \#7b (height $=0.35$ ”).
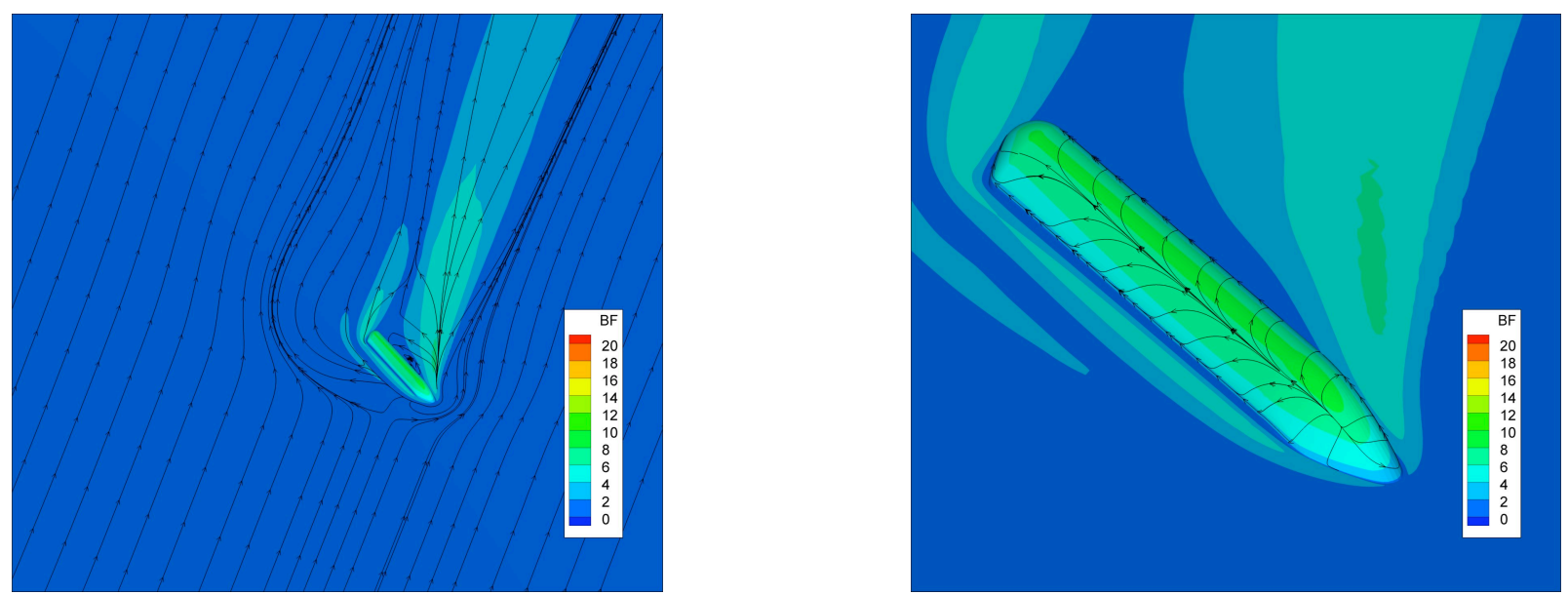

Figure 15. Surface heating BF contours for the Revision \#8b (height = 0.35").

\begin{tabular}{|l|c|c|c|}
\hline \multicolumn{1}{|c|}{ Geometry } & Height (in) & Max protuberance BF & Max acreage BF \\
\hline Initial BLT protuberance & 0.75 & 59 & 15 \\
\hline Revision \#1 & 0.75 & 43 & 14 \\
\hline Revision \#2 & 0.75 & 32 & 12 \\
\hline Revision \#3 & 0.75 & 36 & 10 \\
\hline Revision \#5 & 0.50 & 13 & 7.4 \\
\hline Revision \#6 & 0.50 & 14 & 7.1 \\
\hline Revision \#7b & 0.35 & 8.8 & 5.7 \\
\hline Revision \#8b & 0.35 & 9.1 & 6.3 \\
\hline Revision \#7b & 0.25 & 6.8 & 5.2 \\
\hline
\end{tabular}

Table 2. Comparison of peak heating BF values for the different BLT protuberance revisions. 


\section{Summary of results}

A series of CFD simulations using the DPLR and LAURA codes were computed for the Boundary Layer Transition Flight Experiment in order to estimate the aerothermal environment associated with the installation of the protuberance on the Space Shuttle. Preliminary CFD solutions for a 0.75 " tall protuberance showed significant heating on the trip and the tile surface surround it. As a result, various designs changes were investigated in attempts to lower the peak heating rates. One of the key design parameter in reducing the peak heat flux was to lower the height of the protuberance. Based on the CFD simulations, a BLT protuberance with the Revision \#7b geometry and a trip of 0.25 " was selected for the first flight experiment (installed on STS-119). A second BLT FE with a trip height of 0.35 " was flown on STS-128. The data collected from these experiments are currently being used to reconstruct the thermal response of the protuberance and the heating augmentation downstream of the trip.

\section{Acknowledgments}

Funding for this work was provided by the Orbiter Aeroheating CFD Analysis Program. Chun Tang and Kerry Trumble would like to thank David Saunders (ELORET) for his assistance with the RADIAL_INTERP and FLOW_INTERP utilities.

\section{References}

${ }^{1}$ Anderson, B., "BLT Flight Experiment Overview and In-Situ Measurements," $48^{\text {th }}$ AIAA Aerospace Sciences Meeting, January 4-7, 2010, AIAA Paper 2010-240.

${ }^{2}$ Wood, W. et al., "Comparison of CFD Predictions with Shuttle Global Flight Thermal Imagery and Discrete Surface Measurements," $48^{\text {th }}$ AIAA Aerospace Sciences Meeting, January 4-7, 210, AIAA Paper 2010-0454.

${ }^{3}$ Wright, M., Candler, G., and Bose, D., "Data-Parallel Line Relaxation Method for the Navier-Stokes Equations," AIAA Journal, Vol. 36, No. 9, 1998, pp. 1603-1609.

${ }^{4}$ Gnoffo, P.A., "An Upwind-Biased, point-Implicit relaxation strategies for viscous, hypersonic flows," $9^{\text {th }}$ AIAA Computational Fluid Dynamics Conference, June-13-15, 1989, AIAA-1989-1972.

${ }^{5}$ Gnoffo, P.A., McCandless, R.S., and Yee H.C., "Enhancements To Program LAURA For Computation Of ThreeDimensional Hypersonic Flow," 25th AIAA Aerospace Sciences Meeting, January 12-15, 1987, AIAA-87-0280.

${ }^{6}$ Park, C., "On Convergence of Computation of Chemically Reacting Flows," $23{ }^{\text {rd }}$ AIAA Aerospace Sciences Meeting, January 14-17, 1985, AIAA-85-0247.

${ }^{7}$ Stewart, D. A. and Rakich, J. V., "Catalytic Surface Effects Experiments on Space Shuttle," 16 ${ }^{\text {th }}$ AIAA Thermophysics Conference, June 23-25, 1981, AIAA-81-1143.

${ }^{8}$ Wright, M. J., Loomis, M. P., and Papadopoulos, P. E., "Aerothermal Analysis of the Project Fire II Afterbody Flow," Journal of Thermophysics and Heat Transfer, Vol. 17, No. 2, April-June 2003.

${ }^{9}$ Wright, M. J., Prabhu, D. K., and Martinez, E. R., "Analysis of Apollo Command Module Afterbody Heating Part I: AS202," Journal of Thermophysics and Heat Transfer, Vol. 20, No. 1, January-March 2006.

${ }^{10}$ Tang, C. Y., "Simulations of Afterbody Heating Rates on the Apollo Command Module for AS-202 using Hyperbolic Grids," $9^{\text {th }}$ AIAA/ASME Joint Thermophysics and Heat Transfer Conference, June 5-8, 2006, AIAA-2006-3393.

${ }^{11}$ Tang, C. Y. and Wright, M. J., "Analysis of the Forebody Aeroheating Environment during Genesis Sample Return Capsule Reentry," 45 ${ }^{\text {th }}$ AIAA Aerospace Sciences Meeting, Jan 8-11, 2007, AIAA-2007-1207.

${ }^{12}$ Cheatwood, F. M., Merski, N. R., Riley, C. J., and Mitcheltree, R. A., "Aerothermodynamic Environment Definition for the Genesis Sample Return Capsule," 35 ${ }^{\text {th }}$ AIAA Thermophysics Conference, June 11-14, 2001, AIAA-2001-2889.

${ }^{13}$ Tang, C. Y., Saunders, D. A., Trumble, K. A., and Driver, D. M., "Rapid Aerothermal Simulations of Damage and Repair during a Space Shuttle Mission," $48^{\text {th }}$ AIAA/ASME/ASCE/AHS/ASC Structures, Structural Dynamics, and Materials Conference, April 23-26, 2007, AIAA-2007-1783.

${ }^{14}$ See Pointwise, Inc. website: http://www.pointwise.com/

${ }^{15}$ RADIAL_INTERP is an in-house grid generation/interpolation tool developed by David Saunders (ELORET Corp.) at NASA Ames Research Center.

${ }^{16}$ FLOW_INTERP is an in-house flow interpolation utility developed by David Saunders (ELORET Corp.) at NASA Ames Research Center.

${ }^{17}$ Wright, M. J., White, T. R., and Mangini, N. L., "Data Parallel Line Relaxation (DPLR) Code User Manual Acadia Version 4.01.1," NASA/TM-2009-215388.

${ }^{18}$ Gnoffo, P.A., "Grid Morphing User Manual - Version 1.0.” Feb. 13, 2006 